\title{
Supplemental protease with phytase and xylanase and cereal grain source affected nutrient digestibility and performance of broilers
}

\author{
K.W. McCafferty ${ }^{1}$, A.F. Moss ${ }^{1}$, N.K. Morgan ${ }^{1 *}$, A.J. Cowieson ${ }^{2}$ and M. Choct ${ }^{1}$ \\ ${ }^{1}$ School of Environmental and Rural Sciences, University of New England, Armidale, NSW, 2351, Australia; ${ }^{2}$ DSM Nutritional \\ Products, Kaiseraugst 4303, Switzerland; nmorga20@une.edu.au
}

Received: 9 July 2021 / Accepted: 1 September 2021

(c) 2021 K.W. McCafferty et al.

RESEARCH ARTICLE

POULTRY

\begin{abstract}
A study was conducted to evaluate the effects of supplemental protease and cereal grain type on nutrient digestibility (jejunum and ileum) and performance of broilers offered diets with reduced amino acid concentrations and supplemental xylanase and phytase. A total of 624 male broiler chicks (Ross 308) were randomly distributed into 48 floor pens ( 13 chicks/pen; $0.07 \mathrm{~m}^{2} /$ bird) and offered one of six dietary treatments with eight replicates per treatment. Dietary treatments were either maize- or wheat-based with a positive control (PC) reference diet, a negative control diet without protease (NC; $60 \mathrm{~g} / \mathrm{kg}$ lower amino acid density than PC), and an NC diet with protease. The reduction in amino acid density affected $(P<0.05)$ nutrient digestibility by varying degrees depending on cereal grain source. At $14 \mathrm{~d}$ of age, cereal grain and protease showed a significant interaction $(P<0.05)$ which affected jejunal and ileal starch digestibility, whereby protease increased digestibility in birds fed wheat-based diets but not in those fed maize-based diets. Cereal grain source affected $(P<0.05)$ nitrogen (jejunum and ileum) and digestible energy (DE; ileum), where birds fed wheat-based diets had higher digestibility than those fed maize-based diets. At $28 \mathrm{~d}$ of age, birds fed wheat-based diets had a higher $(P<0.01)$ jejunal and ileal nitrogen digestibility, whereas protease reduced ileal nitrogen digestion. Protease affected ileal starch digestion in birds fed wheat, but not maize-based diets, resulting in a significant cereal grain $\times$ protease interaction $(P<0.05)$. Wheat-based diets had a higher DE than maize-based diets in both the jejunum and ileum. From 15 to $35 \mathrm{~d}$ of age, cereal grain source $(P<0.05)$ affected performance, whereby broilers offered maize-based diets had better performance than those fed wheat-based diets.
\end{abstract}

Keywords: enzyme, cereal grain, Ross 308, jejunum, ileum

\section{Introduction}

Reducing dietary crude protein can have positive impacts on cost and nitrogen excretion (Corzo et al., 2005; Ferguson et al., 1998; Kerr and Kidd, 1999), but can negatively impact broiler growth performance (Dean et al., 2006; Hernández et al., 2012; Waldroup et al., 2005). In general, dietary protein is most appropriately reduced by replacing protein bound amino acids (AA), usually from soybean meal, with feedgrade AA to maintain minimal requirements. Employing this strategy, crude protein reductions of 20 to $30 \mathrm{~g} / \mathrm{kg}$ can be achieved (Greenhalgh et al., 2020). However, reducing both crude protein and amino acid concentrations can further decrease diet cost.

Reducing protein increases dietary dependence on cereal grain proteins. Cereal grain proteins are quantitatively and qualitatively inferior to those from other ingredients (Bewley and Black, 2013). Globally, cereal grains, such as maize and wheat, are the primary energy contributing ingredients in broiler diets (Barletta, 2011). In addition, maize and wheat can provide approximately 200 to $350 \mathrm{~g} /$ $\mathrm{kg}$ of the crude protein in a broiler starter diet. Therefore, protein quality and amino acid (AA) balance of cereal 
grains becomes more critical as dietary crude protein decreases.

Supplementing feed with a mono-component protease enzyme may promote a more efficient release of nutrients, improving the digestibility of protein to account for reductions in amino acid density. Inclusion of protease has been observed to increase AA digestibility by about 3.74\% (Cowieson and Roos, 2013) and responses have been more pronounced in young birds offered diets with reduced nutrient densities (Angel et al., 2011; Cowieson and Roos, 2016; Wang et al., 2008). In the context of the commercial industry, the efficacy of exogenous protease as a stand-alone feed enzyme is not relevant, because most poultry diets are typically formulated with both a phytase and a xylanase. Therefore, the objective of the following experiment was to evaluate the effects of protease supplementation, in combination with xylanase and phytase, and cereal grain source on nutrient digestibility and performance of broiler chicks fed diets formulated with reduced amino acid concentrations.

\section{Materials and methods}

\section{Husbandry}

This experiment was approved by the University of New England Animal Ethics Committee (19-083), which complies with the Australian Code of Practice for the Care and Use of Animal for Scientific Purposes (NHMR, 2013). At hatch, 624 unvaccinated male Ross 308 chicks were obtained from a commercial hatchery (Aviagen, Goulburn, NSW, Australia). Chicks were randomly distributed into 48 floor pens ( 13 chicks per pen; $0.07 \mathrm{~m}^{2}$ per bird) with fresh wood shavings across two environmentally controlled rooms. Each pen was equipped with a hanging pan-feeder and Lubing drinker cups and birds had ad libitum access to feed and water throughout the experiment. Room temperature initially was set at $34{ }^{\circ} \mathrm{C}$ and was gradually reduced as the birds aged, with a final set point of $24{ }^{\circ} \mathrm{C}$ at $35 \mathrm{~d}$ of age. Photoperiod was set at 23L: 1D from 1 to $6 \mathrm{~d}$ of age, and then 20L: 4D from 7 to $35 \mathrm{~d}$ of age.

\section{Dietary treatments}

Prior to diet formulation, representative subsamples of the maize, wheat, and soybean meal were analysed by near-infrared spectroscopy to predict proximates, amino acid (AA) concentrations (AMINO NIR calibrations, Evonik Nutrition \& Care, Hanau, Germany) and apparent metabolisable energy (AME; Precise Nutritional Evaluation calibrations, Adisseo, Commentry, France). Six dietary treatments were provided in a three-phase feeding program from 1 to $14 \mathrm{~d}$ of age (Table 1), 15 to $28 \mathrm{~d}$ of age (Table 2), and 29 to $35 \mathrm{~d}$ of age (Table 3 ).
Treatments (TRT) were of either maize- (TRT1 to 3) or wheat-based (TRT4 to 6). The three dietary TRT for cereal grain source consisted of a positive control (PC) reference diet and two negative control diets (AA density reduced 60 $\mathrm{g} / \mathrm{kg}$ below PC diet) with supplemental protease included at either 0 (NC) or $15,000 \mathrm{PROT} / \mathrm{kg}(\mathrm{NC}+$ Protease). The PC diets (TRT3 and 6) were formulated to be adequate in essential nutrients, but digestible AA $(56,54$, and $91 \mathrm{~g} / \mathrm{kg}$; starter, grower, and finisher, respectively) and AME $(0.2 \mathrm{MJ} / \mathrm{kg}$; starter, grower, and finisher) concentrations were lower than the primary breeder guidelines (Ross, 2014). A mono-component serine protease (RONOZYME ProAct CT; 75,000 PROT/g; DSM Nutritional Products, Kaiseraugst, Switzerland) was included in the supplemented treatments to achieve the feed activity concentrations of $15,000 \mathrm{PROT} / \mathrm{kg}$. No matrix value was applied to supplemental protease. Basal diets were formulated with $0.3 \mathrm{~g} / \mathrm{kg}$ washed builder's sand and protease was included in the supplemented treatments at its expense (e.g. $0.2 \mathrm{~g} / \mathrm{kg}$ protease $+0.1 \mathrm{~g} / \mathrm{kg}$ sand). In unsupplemented treatments $0.3 \mathrm{~g} / \mathrm{kg}$ sand was included. All diets were formulated to contain a mono-component phytase (RONOZYME HIPhos GT; 10,000 FYT/g) and xylanase (RONOZYME WX CT; $1000 \mathrm{FXU} / \mathrm{g}$ ) to achieve feed activity concentrations of 2,000 $\mathrm{FYT} / \mathrm{kg}$ and $200 \mathrm{FXU} / \mathrm{kg}$, respectively (DSM Nutritional Products, Kaiseraugst, Switzerland). In the formulation, phytase was formulated to provide $1.5 \mathrm{~g} / \mathrm{kg}$ of both $\mathrm{Ca}$ and $\mathrm{P}$, respectively. However, no matrix value was applied to xylanase.

Diets were cold-pelleted $\left(65^{\circ} \mathrm{C}\right)$ through a $3 \mathrm{~mm}$ die. Feed form consisted of crumbles during the starter phase and pellets during the grower and finisher phases. A commercial laboratory determined the phytase, xylanase, and protease activity concentrations of all experimental treatments (DSM Nutritional Products Australia Pty Ltd, Wagga Wagga, Australia). The analysed protease, phytase, and xylanase activity concentrations of the starter, grower, and finisher diets are displayed in Table 4.

\section{Measurements}

Birds and feed were weighed at 1, 14, 28, and $35 \mathrm{~d}$ of age to determine body weight (BW), weight gain (BWG), feed intake (FI), and feed conversion ratio (FCR). Mortality was recorded daily and used to adjust FCR based on a birdday basis. The starter period ( 1 to $14 \mathrm{~d}$ of age) was used as an adaptation period, because, upon arrival, the oneday-old chicks weighed less than expected and appeared unthrifty. The chicks were subjected to an extended transportation time and were unvaccinated, which likely affected early health and performance results. After $7 \mathrm{~d}$ of age, birds appeared to recover, because activity and feeding behaviour increased, but initial variability was still evident. Therefore, BW (14 d) and FI (1 to 14 d of age) 
Table 1. Ingredient and nutrient composition of basal starter diets fed to Ross $\times$ Ross 308 male broilers from 1 to $14 \mathrm{~d}$ of age. ${ }^{1}$

\begin{tabular}{|c|c|c|c|c|}
\hline \multirow[t]{2}{*}{ Ingredient, g/kg 'as-fed' } & \multicolumn{2}{|c|}{ Positive control } & \multicolumn{2}{|c|}{ Negative control } \\
\hline & Maize & Wheat & Maize & Wheat \\
\hline Maize (9.5\% CP; 3,150 AME) & 608.5 & na & 639.9 & na \\
\hline Wheat (16.1\% CP; 2,620 AME) & na & 610.6 & na & 642.0 \\
\hline Soybean meal (47.0\% CP; 2,132 AME) & 311.2 & 263.5 & 284.5 & 234.3 \\
\hline Canola oil & 37.3 & 82.6 & 33 & 80.7 \\
\hline Calcium carbonate & 10.7 & 11.0 & 10.9 & 11.2 \\
\hline Dicalcium phosphate & 10.0 & 9.2 & 10.0 & 9.2 \\
\hline Sodium chloride & 1.9 & 1.6 & 1.9 & 1.6 \\
\hline Sodium bicarbonate & 1.9 & 2.5 & 2 & 2.6 \\
\hline Vitamin premix ${ }^{2}$ & 0.9 & 0.9 & 0.9 & 0.9 \\
\hline Mineral premix ${ }^{3}$ & 1.0 & 1.0 & 1.0 & 1.0 \\
\hline Choline chloride & 3.8 & 3.8 & 3.8 & 3.8 \\
\hline L-Lys.HCl & 3.0 & 3.8 & 2.9 & 3.7 \\
\hline DL-Met & 3.1 & 2.5 & 2.8 & 2.1 \\
\hline L-Thr & 1.0 & 1.3 & 0.9 & 1.1 \\
\hline Phytase $^{4}$ & 0.2 & 0.2 & 0.2 & 0.2 \\
\hline Xylanase $^{4}$ & 0.2 & 0.2 & 0.2 & 0.2 \\
\hline Sand $^{5}$ & 0.3 & 0.3 & 0.3 & 0.3 \\
\hline Titanium dioxide & 5.0 & 5.0 & 5.0 & 5.0 \\
\hline \multicolumn{5}{|c|}{ Calculated nutrient content ( $\mathrm{g} / \mathrm{kg}$, unless otherwise indicated) } \\
\hline $\mathrm{AME}, \mathrm{MJ} / \mathrm{kg}$ & 12.35 & 12.35 & 12.35 & 12.35 \\
\hline Starch & 396.8 & 370.4 & 417.0 & 389.2 \\
\hline Crude protein & 209.5 & 228.3 & 199.8 & 219.2 \\
\hline SID $^{6}$ Lys & 11.8 & 11.8 & 11.1 & 11.1 \\
\hline SID Met & 5.9 & 5.3 & 5.4 & 4.8 \\
\hline SID TSAA & 8.6 & 8.6 & 8.1 & 8.1 \\
\hline SID Thr & 7.6 & 7.6 & 7.1 & 7.1 \\
\hline SID Val & 8.7 & 8.8 & 8.3 & 8.4 \\
\hline SID Arg & 12.2 & 12.2 & 11.4 & 11.4 \\
\hline SID Trp & 2.1 & 2.5 & 2.0 & 2.4 \\
\hline $\mathrm{Ca}$ & 9.0 & 9.0 & 9.0 & 9.0 \\
\hline Non-phytate P & 4.5 & 4.5 & 4.5 & 4.5 \\
\hline $\mathrm{Na}$ & 1.8 & 1.8 & 1.8 & 1.8 \\
\hline
\end{tabular}

${ }^{1} \mathrm{AME}=$ apparent metabolisable energy; $\mathrm{CP}=$ crude protein; na = not analysed.

2 Vitamin premix supplied per kilogram of diet: retinol, $12,000 \mathrm{IU}$; cholecalciferol, $5,000 \mathrm{IU}$; tocopheryl acetate, $75 \mathrm{mg}$, menadione, $3 \mathrm{mg}$; thiamine, $3 \mathrm{mg}$; riboflavin, $8 \mathrm{mg}$; niacin, $55 \mathrm{mg}$; pantothenate, $13 \mathrm{mg}$; pyridoxine, $5 \mathrm{mg}$; folate, $2 \mathrm{mg}$; cyanocobalamin, $16 \mu \mathrm{\mu g}$; biotin, $200 \mu \mathrm{g}$; cereal-based carrier, $149 \mathrm{mg}$; mineral oil, $2.5 \mathrm{mg}$.

${ }^{3}$ Trace mineral premix supplied per kilogram of diet: $\mathrm{Cu}$ (sulphate), $16 \mathrm{mg}$; Fe (sulphate), $40 \mathrm{mg}$; I (iodide), $1.25 \mathrm{mg}$; Se (selenate), $0.3 \mathrm{mg} ; \mathrm{Mn}$ (sulphate and oxide), $120 \mathrm{mg}$; Zn (sulphate and oxide), $100 \mathrm{mg}$; cereal-based carrier, $128 \mathrm{mg}$; mineral oil, $3.75 \mathrm{mg}$.

${ }^{4}$ RONOZYME ${ }^{\circledR}$ HiPhos and RONOZYME ${ }^{\circledR}$ WX were used as sources of phytase and xylanase, respectively. A phytase matrix value of $1.5 \mathrm{~g} / \mathrm{kg} \mathrm{Ca}$ and $1.5 \mathrm{~g} / \mathrm{kg}$ digestible $P$ was used. No energy matrix was applied to xylanase.

${ }^{5}$ Sand was used as the variable portion. RONOZYME ${ }^{\circledR}$ ProAct was included at 0 or $200 \mathrm{mg} / \mathrm{kg}$ to achieve activity protease activity concentrations of 0 or 15,000 $\mathrm{PROT} / \mathrm{kg}$, respectively, in each of the 2 basal NC diets to achieve the supplemented treatments.

${ }^{6} \mathrm{SID}=$ standardised ileal digestibility values. Dies were formulated on digestible amino acid basis using SID values predicted by AMINOßNIR (Evonik Nutrition \& Care, Hanua, Germany).

were included as covariates into the mixed-effects model to adjust performance data ( 15 to $35 \mathrm{~d}$ of age).

\section{Apparent jejunal and ileal digestibility}

At 14 and $28 \mathrm{~d}$ of age, four or three birds per pen, respectively, were randomly selected, weighed, and euthanised via cervical dislocation for jejunal and ileal digesta contents. Digesta contents from the entire jejunum (end of duodenal loop to Meckel's diverticulum) and ileum (Meckel's diverticulum to ileo-caecal junction) were gently squeezed into polypropylene cups. Samples were pooled per cage and frozen at $-20{ }^{\circ} \mathrm{C}$ until further analysis. Apparent jejunal and ileal digestibility coefficients (DC) of nitrogen, starch, and energy were calculated on a DM basis with the following equation:
Apparent DC $(\%)=\frac{\frac{\text { Nutrient }}{\mathrm{TiO}_{2}} \text { diet }-\frac{\text { Nutrient }}{\mathrm{TiO}_{2}} \text { digesta }}{\frac{\text { Nutrient }}{\mathrm{TiO}_{2}} \text { diet }}$

where nutrient corresponded to nitrogen, starch, or GE. Apparent digestible energy (DE) was calculated by the following equation:

Apparent DE $\left(\frac{\mathrm{MJ}}{\mathrm{kg}}\right)=\mathrm{GE}_{\text {diet }} \times($ Apparent DC of energy $)$

\section{Chemical analyses}

Diet and digesta samples were freeze dried (Christ Alpha 1-2LDplus Freeze Dryer, Martin Christ Gefriertrocknungsanlagen, Osterode, Germany). Dried samples were ground 
Table 2. Ingredient and nutrient composition of basal grower diets fed to Ross $\times$ Ross 308 male broilers from 15 to $28 \mathrm{~d}$ of age. 1

\begin{tabular}{|c|c|c|c|c|}
\hline \multirow[t]{2}{*}{ Ingredient, g/kg 'as-fed' } & \multicolumn{2}{|c|}{ Positive control } & \multicolumn{2}{|c|}{ Negative control } \\
\hline & Maize & Wheat & Maize & Wheat \\
\hline Maize $(9.5 \%$ CP; 3,150 AME) & 642.9 & na & 669.7 & na \\
\hline Wheat (16.1\% CP; $2,620 \mathrm{AME})$ & na & 645.1 & na & 672.0 \\
\hline Soybean meal (47.0\% CP; $2,132 \mathrm{AME})$ & 267.7 & 217.3 & 244.8 & 192.3 \\
\hline Canola oil & 46.9 & 94.8 & 43.2 & 93.1 \\
\hline Calcium carbonate & 10.9 & 11.3 & 11.0 & 11.4 \\
\hline Dicalcium phosphate & 10.2 & 9.4 & 10.3 & 9.4 \\
\hline Sodium chloride & 1.9 & 1.7 & 2.0 & 1.7 \\
\hline Sodium bicarbonate & 2.0 & 2.6 & 2.0 & 2.6 \\
\hline Vitamin premix ${ }^{2}$ & 0.9 & 0.9 & 0.9 & 0.9 \\
\hline Mineral premix ${ }^{3}$ & 1.0 & 1.0 & 1.0 & 1.0 \\
\hline Choline chloride & 3.8 & 3.8 & 3.8 & 3.8 \\
\hline L-Lys·HCl & 2.8 & 3.6 & 2.7 & 3.5 \\
\hline DL-Met & 2.6 & 1.9 & 2.3 & 1.6 \\
\hline L-Thr & 0.8 & 1.1 & 0.7 & 1.0 \\
\hline Phytase 4 & 0.2 & 0.2 & 0.2 & 0.2 \\
\hline Xylanase $^{4}$ & 0.2 & 0.2 & 0.2 & 0.2 \\
\hline Sand $^{5}$ & 0.3 & 0.3 & 0.3 & 0.3 \\
\hline Titanium dioxide & 5.0 & 5.0 & 5.0 & 5.0 \\
\hline \multicolumn{5}{|c|}{ Calculated nutrient content ( $\%$, unless otherwise indicated) } \\
\hline $\mathrm{AME}, \mathrm{kcal} / \mathrm{kg}$ & 12.76 & 12.76 & 12.76 & 12.76 \\
\hline Starch & 418.8 & 390.9 & 436.1 & 407.0 \\
\hline Crude protein & 191.7 & 210.9 & 183.1 & 203.1 \\
\hline SID $^{6}$ Lys & 10.6 & 10.6 & 10.0 & 10.0 \\
\hline SID Met & 4.5 & 4.5 & 4.2 & 4.1 \\
\hline SID TSAA & 7.7 & 7.7 & 7.3 & 7.3 \\
\hline SID Thr & 6.8 & 6.8 & 6.4 & 6.4 \\
\hline SID Val & 8.0 & 8.1 & 7.7 & 7.8 \\
\hline SID Arg & 10.9 & 10.9 & 10.3 & 10.3 \\
\hline SID Trp & 1.9 & 2.3 & 1.8 & 2.2 \\
\hline $\mathrm{Ca}$ & 9.0 & 9.0 & 9.0 & 9.0 \\
\hline Non-phytate P & 4.5 & 4.5 & 4.5 & 4.5 \\
\hline $\mathrm{Na}$ & 1.9 & 1.7 & 2.0 & 1.7 \\
\hline
\end{tabular}

${ }^{1} \mathrm{AME}=$ apparent metabolisable energy; $\mathrm{CP}=$ crude protein; na = not analysed.

2 Vitamin premix supplied per kilogram of diet: retinol, $12,000 \mathrm{IU}$; cholecalciferol, $5,000 \mathrm{IU}$; tocopheryl acetate, $75 \mathrm{mg}$, menadione, $3 \mathrm{mg}$; thiamine, $3 \mathrm{mg}$; riboflavin, $8 \mathrm{mg}$; niacin, $55 \mathrm{mg}$; pantothenate, $13 \mathrm{mg}$; pyridoxine, $5 \mathrm{mg}$; folate, $2 \mathrm{mg}$; cyanocobalamin, $16 \mu \mathrm{g}$; biotin, $200 \mu \mathrm{g}$; cereal-based carrier, $149 \mathrm{mg}$; mineral oil, $2.5 \mathrm{mg}$.

${ }^{3}$ Trace mineral premix supplied per kilogram of diet: $\mathrm{Cu}$ (sulphate), $16 \mathrm{mg}$; Fe (sulphate), $40 \mathrm{mg}$; I (iodide), $1.25 \mathrm{mg}$; Se (selenate), $0.3 \mathrm{mg} ; \mathrm{Mn}$ (sulphate and oxide), $120 \mathrm{mg}$; Zn (sulphate and oxide), $100 \mathrm{mg}$; cereal-based carrier, $128 \mathrm{mg}$; mineral oil, $3.75 \mathrm{mg}$.

${ }^{4}$ RONOZYME ${ }^{\circledR}$ HiPhos and RONOZYME ${ }^{\circledR}$ WX were used as sources of phytase and xylanase, respectively. A phytase matrix value of $1.5 \mathrm{~g} / \mathrm{kg} \mathrm{Ca}$ and $1.5 \mathrm{~g} / \mathrm{kg}$ digestible $P$ was used. No energy matrix was applied to xylanase.

${ }^{5}$ Sand was used as the variable portion. RONOZYME ${ }^{\circledR}$ ProAct was included at 0 or $200 \mathrm{mg} / \mathrm{kg}$ to achieve activity protease activity concentrations of 0 or 15,000 $\mathrm{PROT} / \mathrm{kg}$, respectively, in each of the 2 basal NC diets to achieve the supplemented treatments.

${ }^{6} \mathrm{SID}=$ standardised ileal digestibility values. Diets were formulated on digestible amino acid basis using SID values predicted by AMINO ${ }^{\circledR}$ NIR (Evonik Nutrition \& Care, Hanua, Germany).

to pass through a $0.5 \mathrm{~mm}$ screen and samples were analysed for nitrogen, starch, gross energy (GE). Dry matter of wet samples was determined using a forced air oven at $105^{\circ} \mathrm{C}$ for $12 \mathrm{~h}$. Nitrogen content was determined by the Dumas combustion method (method 990.03; (AOAC, 2005) with a Leco FP-200 N analyser (Leco Corp., St. Joseph, MI, USA) using N correction factor of 6.25 for crude protein (CP) determination. Starch concentration was determined enzymatically using Megazyme Total Starch Assay Kit (Megazyme Int., Wicklow, Ireland). Gross energy was determined using a 6400 automatic isoperibol oxygen bomb calorimeter (Parr Instruments, Moline, IA, USA). Additionally, diets and digesta were analysed for titanium dioxide $\left(\mathrm{TiO}_{2}\right)$ concentrations in quadruplicate and duplicate replicates, respectively, by the method described by Short et al. (1996).

\section{Statistical analysis}

Data were arranged in a randomised complete block design structure with pen location as the blocking factor with eight replicate pens per treatment. Pen was considered the experimental unit. Two statistical models were employed to conduct analyses. Additionally, to account for the unexpected variation observed during the starter period, BW (14 d) and FI (1 to $14 \mathrm{~d}$ of age) were included as covariates model to adjust performance data. A twoway ANOVA in PROC MIXED (SAS, 2015) was used to evaluate the interactive and main effects of cereal grain source (maize- or wheat-based) and protease inclusion $(0$ or $15,000 \mathrm{PROT} / \mathrm{kg}$ ) on nutrient digestibility and growth performance. Additionally, a one-way ANOVA in PROC MIXED (SAS, 2015) with pre-planned orthogonal contrasts was used to assess the effects on nutrient digestibility and 
Table 3. Ingredient and nutrient composition of basal finisher diets fed to Ross $\times$ Ross 308 male broilers from 29 to $35 \mathrm{~d}$ of age. ${ }^{1}$

\begin{tabular}{|c|c|c|c|c|}
\hline \multirow[t]{2}{*}{ Ingredient, g/kg 'as-fed' } & \multicolumn{2}{|c|}{ Positive control } & \multicolumn{2}{|c|}{ Negative control } \\
\hline & Maize & Wheat & Maize & Wheat \\
\hline Maize (9.5\% CP; 3,150 AME) & 682.4 & na & 704.7 & na \\
\hline Wheat (16.1\% CP; 2,620 AME) & na & 684.7 & na & 707.0 \\
\hline Soybean meal (47.0\% CP; 2,132 AME) & 226.9 & 173.4 & 207.8 & 152.5 \\
\hline Canola oil & 48.6 & 99.4 & 45.5 & 98.0 \\
\hline Calcium carbonate & 11.1 & 11.4 & 11.2 & 11.6 \\
\hline Dicalcium phosphate & 10.4 & 9.5 & 10.4 & 9.6 \\
\hline Sodium chloride & 2.0 & 1.7 & 2.0 & 1.7 \\
\hline Sodium bicarbonate & 2.0 & 2.6 & 2.1 & 2.7 \\
\hline Vitamin premix ${ }^{2}$ & 0.9 & 0.9 & 0.9 & 0.9 \\
\hline Mineral premix ${ }^{3}$ & 1.0 & 1.0 & 1.0 & 1.0 \\
\hline Choline chloride & 3.8 & 3.8 & 3.8 & 3.8 \\
\hline L-Lys.HCl & 2.6 & 3.5 & 2.5 & 3.4 \\
\hline DL-Met & 2.1 & 1.4 & 1.8 & 1.2 \\
\hline L-Thr & 0.6 & 0.9 & 0.5 & 0.8 \\
\hline Phytase 4 & 0.2 & 0.2 & 0.2 & 0.2 \\
\hline Xylanase $^{4}$ & 0.2 & 0.2 & 0.2 & 0.2 \\
\hline Sand $^{5}$ & 0.3 & 0.3 & 0.3 & 0.3 \\
\hline Titanium dioxide & 5.0 & 5.0 & 5.0 & 5.0 \\
\hline \multicolumn{5}{|c|}{ Calculated nutrient content ( $\%$, unless otherwise indicated) } \\
\hline AME, MJ/kg & 12.97 & 12.97 & 12.97 & 12.97 \\
\hline Starch & 444.2 & 414.6 & 458.6 & 428.0 \\
\hline Crude protein & 175.6 & 191.6 & 168.5 & 189.6 \\
\hline SID $^{6}$ Lys & 9.5 & 9.5 & 9.0 & 9.0 \\
\hline SID Met & 4.5 & 3.8 & 4.2 & 3.6 \\
\hline SID TSAA & 7.0 & 7.0 & 6.6 & 6.7 \\
\hline SID Thr & 6.1 & 6.1 & 5.8 & 5.8 \\
\hline SID Val & 7.4 & 7.5 & 7.1 & 7.2 \\
\hline SID Arg & 9.8 & 9.8 & 9.3 & 9.3 \\
\hline SID Trp & 1.7 & 2.1 & 1.6 & 2.0 \\
\hline $\mathrm{Ca}$ & 9.0 & 9.0 & 9.0 & 9.0 \\
\hline Non-phytate P & 4.5 & 4.5 & 4.5 & 4.5 \\
\hline $\mathrm{Na}$ & 1.8 & 1.8 & 1.8 & 1.8 \\
\hline
\end{tabular}

${ }^{1} \mathrm{AME}=$ apparent metabolisable energy; $\mathrm{CP}=$ crude protein; na = not analysed.

2 Vitamin premix supplied per kilogram of diet: retinol, $12,000 \mathrm{IU}$; cholecalciferol, $5,000 \mathrm{IU}$; tocopheryl acetate, $75 \mathrm{mg}$, menadione, $3 \mathrm{mg}$; thiamine, $3 \mathrm{mg}$; riboflavin, $8 \mathrm{mg}$; niacin, $55 \mathrm{mg}$; pantothenate, $13 \mathrm{mg}$; pyridoxine, $5 \mathrm{mg}$; folate, $2 \mathrm{mg}$; cyanocobalamin, $16 \mu \mathrm{\mu g}$; biotin, $200 \mu \mathrm{g}$; cereal-based carrier, $149 \mathrm{mg}$; mineral oil, $2.5 \mathrm{mg}$.

${ }^{3}$ Trace mineral premix supplied per kilogram of diet: $\mathrm{Cu}$ (sulphate), $16 \mathrm{mg}$; Fe (sulphate), $40 \mathrm{mg}$; I (iodide), $1.25 \mathrm{mg}$; Se (selenate), $0.3 \mathrm{mg}$; Mn (sulphate and oxide), $120 \mathrm{mg}$; Zn (sulphate and oxide), $100 \mathrm{mg}$; cereal-based carrier, $128 \mathrm{mg}$; mineral oil, $3.75 \mathrm{mg}$.

${ }^{4}$ RONOZYME $^{\circledR}$ HiPhos and RONOZYME ${ }^{\circledR}$ WX were used as sources of phytase and xylanase, respectively. A phytase matrix value of $1.5 \mathrm{~g} / \mathrm{kg}$ Ca and $1.5 \mathrm{~g} / \mathrm{kg}$ digestible $P$ was used. No energy matrix was applied to xylanase.

${ }^{5}$ Sand was used as the variable portion. RONOZYME ${ }^{\circledR}$ ProAct was included at 0 or $200 \mathrm{mg} / \mathrm{kg}$ to achieve activity protease activity concentrations of 0 or 15,000 $\mathrm{PROT} / \mathrm{kg}$, respectively, in each of the 2 basal NC diets to achieve the supplemented treatments.

${ }^{6} \mathrm{SID}=$ standardised ileal digestibility values. Diets were formulated on digestible amino acid basis using SID values predicted by AMINO ${ }^{\circledR}$ NIR (Evonik Nutrition \& Care, Hanua, Germany).

growth performance between those offered the PC and NC diets. Statistical significance was established at $P \leq 0.05$, and a trend was considered at $P \leq 0.10$ ). Interactive and main effects were separated using Tukey's honestly significantly different test.

\section{Results}

\section{Performance}

The starter period ( 1 to $14 \mathrm{~d}$ of age) was used as an adaptation period, because upon arrival day-old chicks exhibited a low BW (35 g) and appeared unthrifty. After $7 \mathrm{~d}$ of age, birds began to be more active and feeding behaviour improved, indicating that the initial stress was being resolved. From 1 to $14 \mathrm{~d}$ of age, the average BW,
BWG, FI and FCR were 347 g, 311 g, 467 g, and 1.509, respectively. However, initial variability was still evident. Consequently, BW (14 d) and FI (1 to 14 d of age) were included as covariates into the mixed-effects model to adjust performance data and only the 15 to $35 \mathrm{~d}$ of age performance data was analysed and reported.

From 15 to $35 \mathrm{~d}$ of age, no interactions or protease main effects $(P>0.05)$ on broiler growth performance were observed (Table 5$)$. Cereal grain main effects $(P<0.05)$ on final BW, BWG, FI and FCR were observed. Broilers offered the maize-based diets had 20.0,11.7, 16.9\% higher $35 \mathrm{~d} \mathrm{BW}$ $(P=0.001)$, BWG $(P=0.002)$ and FI $(P=0.001)$, respectively, and $6.7 \%$ lower FCR $(P=0.05)$ than those offered wheatbased diets. Broilers offered the maize-based PC tended to have $5.0 \%$ higher $35 \mathrm{~d} \mathrm{BW}(P=0.09)$ than those offered 
Table 4. Analysed phytase, xylanase, and protease activity concentrations of the starter (1 to $14 \mathrm{~d}$ of age), grower (15 to $28 \mathrm{~d}$ of age), and finisher (29 to $35 \mathrm{~d}$ of age) diets. ${ }^{1}$

\begin{tabular}{|c|c|c|c|c|c|c|c|c|c|}
\hline \multirow[t]{2}{*}{ Diet treatment } & \multicolumn{3}{|c|}{1 to $14 \mathrm{~d}$ of age } & \multicolumn{3}{|c|}{15 to $28 \mathrm{~d}$ of age } & \multicolumn{3}{|c|}{29 to $35 \mathrm{~d}$ of age } \\
\hline & $\begin{array}{l}\text { Protease } \\
\text { (PROT/kg) }^{2}\end{array}$ & $\begin{array}{l}\text { Xylanase } \\
\text { (FXU/kg) }^{3}\end{array}$ & $\begin{array}{l}\text { Phytase } \\
\text { (FYT/kg) }^{4}\end{array}$ & $\begin{array}{l}\text { Protease } \\
\text { (PROT/kg) }\end{array}$ & $\begin{array}{l}\text { Xylanase } \\
\text { (FXU/kg) }\end{array}$ & $\begin{array}{l}\text { Phytase } \\
\text { (FYT/kg) }\end{array}$ & $\begin{array}{l}\text { Protease } \\
\text { (PROT/kg) }\end{array}$ & $\begin{array}{l}\text { Xylanase } \\
\text { (FXU/kg) }\end{array}$ & $\begin{array}{l}\text { Phytase } \\
\text { (FYT/kg) }\end{array}$ \\
\hline Maize NC & $n a^{5}$ & 133 & 2,535 & na & 210 & 2,365 & na & 185 & 2,181 \\
\hline Maize NC + protease & 14,020 & 218 & 2,185 & 13,930 & 159 & 2,172 & 11,900 & 176 & 2,548 \\
\hline Maize PC & na & 244 & 2,719 & na & 164 & 1,970 & na & 208 & 2,495 \\
\hline Wheat NC & na & 144 & 3,158 & na & 133 & 2,901 & na & 89 & 2,648 \\
\hline Wheat PC & na & 119 & 2,819 & na & 111 & 3,124 & na & 104 & 2,656 \\
\hline
\end{tabular}

1 Values represent average of 3 replicates samples. Enzyme activity concentrations were determined by an outside laboratory (DSM Nutritional Products, Wagga Wagga, NSW, Australia)

2 Protease $=$ RONOZYME ProAct (DSM Nutritional Products, Kaiseraugst, Switzerland) which provides 75,000 PROT/g was included in the supplemental treatments at $0.02 \%$ to achieve a protease activity of $15,000 \mathrm{PROT} / \mathrm{kg}$.

${ }^{3}$ Xylanase $=$ RONOZYME WX (DSM Nutritional Products, Kaiseraugst, Switzerland) which provides 1000 FXU/g was included in all supplemented treatments at $0.02 \%$ to achieve a xylanase activity of $200 \mathrm{FXU} / \mathrm{kg}$.

${ }^{4}$ Phytase $=$ RONOZYME HiPhos (DSM Nutritional Products, Kaiseraugst, Switzerland) which provides 10,000 FYT/g was included in all treatments at 0.02\% to achieve a phytase activity of $2,000 \mathrm{FYT} / \mathrm{kg}$.

${ }^{5}$ na $=$ not analysed.

the maize-based NC. Broilers fed the wheat PC had a $6.7 \%$ lower FCR $(P=0.05)$ than those offered the wheat-based NC. Cumulative mortality was $2.2 \%$ and was not $(P>0.05)$ affected by dietary treatments.

\section{Jejunal and ileal digestibility}

At $14 \mathrm{~d}$ of age, cereal grain and protease interacted $(P<0.05)$ to affect jejunal $(P=0.001)$ and ileal $(P=0.006)$ starch DC

Table 5. Growth performance of Ross $\times$ Ross 308 male broilers fed maize- or wheat-based diets without or with supplemental protease from 15 to $35 \mathrm{~d}$ of age. ${ }^{1}$

\begin{tabular}{|c|c|c|c|c|c|}
\hline Cereal grain & Dietary treatment ${ }^{2}$ & $35 \mathrm{~d}$ Body weight (kg) & Body weight gain $(\mathrm{kg})$ & Feed intake (kg) & $\mathrm{FCR}^{3}(\mathrm{~kg} / \mathrm{kg})$ \\
\hline Maize & $\begin{array}{l}\text { NC } \\
\text { NC + protease }{ }^{4} \\
\text { PC }\end{array}$ & $\begin{array}{l}2.051 \\
2.068 \\
2.154\end{array}$ & $\begin{array}{l}1.705 \\
1.721 \\
1.800\end{array}$ & $\begin{array}{l}2.425 \\
2.478 \\
2.464\end{array}$ & $\begin{array}{l}1.421 \\
1.442 \\
1.380\end{array}$ \\
\hline $\begin{array}{l}\text { Wheat } \\
\text { SEM } \\
\text { Cereal grain main effects }\end{array}$ & $\begin{array}{l}\mathrm{NC} \\
\mathrm{NC} \text { + protease } \\
\mathrm{PC}\end{array}$ & $\begin{array}{l}1.752 \\
1.694 \\
1.846 \\
0.051\end{array}$ & $\begin{array}{l}1.405 \\
1.347 \\
1.492 \\
0.051\end{array}$ & $\begin{array}{l}2.130 \\
2.063 \\
2.122 \\
0.055\end{array}$ & $\begin{array}{l}1.532 \\
1.540 \\
1.429 \\
0.042\end{array}$ \\
\hline $\begin{array}{l}\text { Maize } \\
\text { Wheat } \\
\text { SEM }\end{array}$ & & $\begin{array}{l}2.060 \\
1.723 \\
0.040\end{array}$ & $\begin{array}{l}1.713 \\
1.376 \\
0.040\end{array}$ & $\begin{array}{l}2.451 \\
2.097 \\
0.044\end{array}$ & $\begin{array}{l}1.432 \\
1.536 \\
0.033\end{array}$ \\
\hline $\begin{array}{l}\text { Protease main effects } \\
\text { Analysis of variance }\end{array}$ & $\begin{array}{l}\mathrm{NC} \\
\mathrm{NC}+\text { protease } \\
\mathrm{SEM}\end{array}$ & $\begin{array}{l}1.902 \\
1.881 \\
0.032\end{array}$ & $\begin{array}{l}1.555 \\
1.534 \\
0.032 \\
\text { Probabilities }\end{array}$ & $\begin{array}{l}2.277 \\
2.271 \\
0.036\end{array}$ & $\begin{array}{l}1.477 \\
1.491 \\
0.028\end{array}$ \\
\hline $\begin{array}{l}\text { Cereal grain } \times \text { Protease }^{5} \\
\text { Cereal grain } \\
\text { Protease } \\
\text { Pre-planned orthogonal contrasts } \\
\text { Maize NC vs Maize PC } \\
\text { Wheat NC vs Wheat PC }\end{array}$ & $\begin{array}{l}0.46 \\
0.001 \\
0.63 \\
0.09 \\
0.25\end{array}$ & $\begin{array}{l}0.46 \\
0.001 \\
0.63 \\
0.09 \\
0.24\end{array}$ & $\begin{array}{l}0.26 \\
0.001 \\
0.88 \\
0.95 \\
0.57\end{array}$ & $\begin{array}{l}0.87 \\
0.05 \\
0.67 \\
0.12 \\
0.03\end{array}$ & \\
\hline $\begin{array}{l}{ }^{1} \text { Each value represents the least-s } \\
{ }^{2} \text { Dietary treatment consisted of } 6 \mathrm{p} \\
\text { (3) Maize-based positive control (P } \\
{ }^{3} \text { FCR = feed conversion ratio (feed } \\
{ }^{4} \text { RONOZYME }{ }^{\circledR} \text { ProAct (DSM Nutriti } \\
\text { phytase and xylanase. RONOZYME } \\
\text { Ca and } 1.5 \mathrm{~g} / \mathrm{kg} \text { digestible P was us }\end{array}$ & $\begin{array}{l}\text { re means of } 8 \text { repli } \\
\text { ible diets: (1) Maize } \\
\text { (4) Wheat-based N } \\
\text { ake/body weight ga } \\
\text { l Products, Kaisera } \\
\text { tiPhos and RONOZ } \\
\text { No energy matrix }\end{array}$ & $\begin{array}{l}\text { ens with approximately } \\
\text { Whegative control (NC; } \\
\text { Wheat-based NC + } 200 \\
\text { Srected for mortality on } \\
\text { Bitzerland) included at } \\
\text { WX were used as sourc } \\
\text { pplied to xylanase. }\end{array}$ & $\begin{array}{l}9 \text { and } 6 \text { birds per pen fro } \\
\text { amino acid density } 94 \% \text { c } \\
0 \mathrm{mg} / \mathrm{kg} \text { protease; or }(6) \mathrm{V} \\
\text { a bird day basis. } \\
200 \mathrm{mg} / \mathrm{kg} \text { to provide } 15,0 \\
\text { ces of phytase and xylana }\end{array}$ & $\begin{array}{l}\text { If } 15 \text { to } 28 \text { and } 29 \\
\text { of PC); (2) Maize-b } \\
\text { Wheat-based PC. } \\
000 \text { PROT/kg. All di } \\
\text { ase, respectively. A }\end{array}$ & $\begin{array}{l}\mathrm{d} \text { of age, resp } \\
\mathrm{VC}+200 \mathrm{mg} / \mathrm{k} \\
\text { ntained both st } \\
\text { se matrix value }\end{array}$ \\
\hline
\end{tabular}


(Table 6), protease increased digestibility in birds fed wheat-based diets but not those fed maize-based diets. Jejunal starch $\mathrm{DC}$ was highest $(P<0.05)$ in broilers offered the maize-based NC diet, but lowest $(P<0.05)$ in those fed the wheat-based $\mathrm{NC}$ diet. Broilers offered the maizebased NC diet had a $2.8 \%$ higher $(P<0.05)$ jejunal starch DC than those offered the maize-based NC diet containing protease. In contrast, broilers offered the maize-based NC and the wheat-based NC diet with protease had a 5.8\% higher $(P<0.05)$ jejunal starch DC than those offered the wheat-based NC diet. Similarly, broilers offered the wheatbased NC diet with protease had a 1.6\% higher $(P<0.05)$ ileal starch DC than those the wheat-based NC diet, whereas broilers offered the maize-based $\mathrm{NC}$ diet without and with protease had similar DC $(P>0.05)$ compared with the those offered the wheat-based NC diet without and with protease. A cereal grain main effect was observed for jejunal $(P=0.004)$ and ileal $(P=0.001)$ nitrogen DC. Broilers offered wheat-based diets had a $34.5 \%$ and $3.9 \%$ higher $(P<0.05)$ jejunal and ileal nitrogen DC, respectively, than those offered maize-based diets. Likewise, broilers offered wheat-based diets had a $1.7 \%$ higher $(P=0.001) \mathrm{DE}$ than those fed maize-based diets. The reduction in AA density in the maize-based diet affected ileal nitrogen $(P=0.001)$ and jejunal starch $(P=0.009)$ digestibility with broilers receiving the maize-based NC diet having a 2.7 and $2.5 \%$ higher DC, respectively than those offered the maize-based PC. Likewise, a trend was observed for ileal $\mathrm{DE}(P=0.06)$ with broilers receiving the maize-based $\mathrm{PC}$ having $1.9 \%$ higher $(P<0.10)$ ileal DE than those given the maize-based NC. Likewise, the reduction in AA density in the wheat-based diet affected jejunal starch $(P=0.001)$, ileal starch $(P=0.02)$ and ileal $\mathrm{DE}(P=0.05)$, with broilers receiving the $\mathrm{PC}$ having a 4.0, 1.2 and $1.9 \%$ higher $(P<0.05) \mathrm{DC}$, respectively, than those offered the wheat-based $\mathrm{NC}$ diet.

At $28 \mathrm{~d}$ of age, cereal grain and protease interacted to affect ileal starch DC $(P=0.05$; Table 7$)$. Broilers receiving the wheat-based NC diet had a $3.5 \%$ higher $(P<0.05)$ ileal starch DC than those offered the wheat-based NC containing protease. However, broilers offered the maize-based NC diets with and without protease were similar $(P>0.05)$

Table 6. Apparent nitrogen and starch digestibility coefficients and digestible energy in the jejunum and ileum of Ross $\times$ Ross 308 male broiler chicks fed maize- or wheat-based diets without or with supplemental protease at $14 \mathrm{~d}$ of age.,2

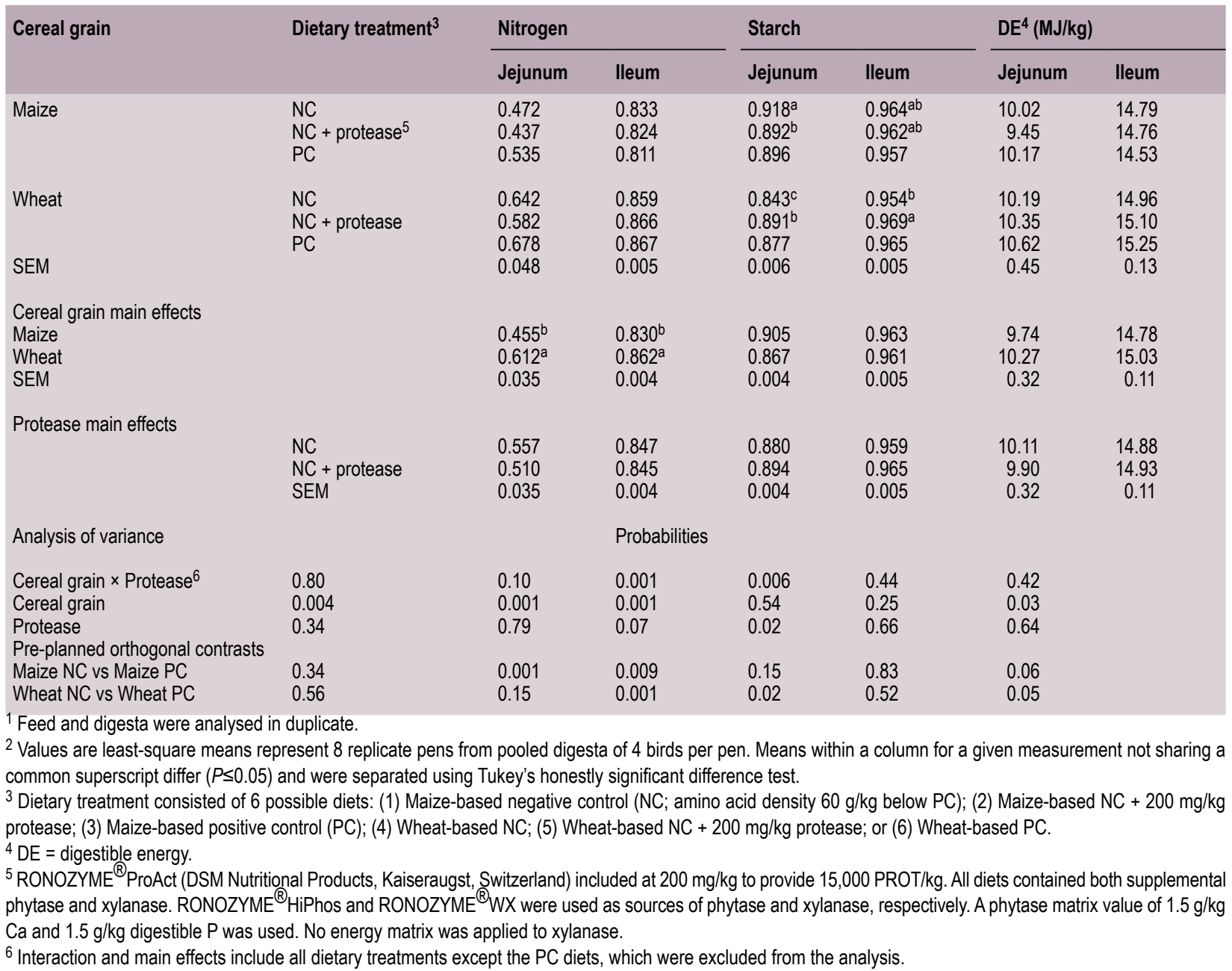


to those offered the wheat-based diets without and with protease. Cereal grain influenced jejunal nitrogen DC $(P=0.002)$, ileal nitrogen DC $(P=0.001)$, jejunal starch DC $(P=0.001)$, jejunal DE $(P=0.02)$ and ileal DE $(P=0.001)$. Broilers receiving wheat-based diets had 15.4 and $5.0 \%$ higher $(P<0.05)$ jejunal and ileal nitrogen $D C$, respectively, than those offered maize-based diets. Similarly, broilers offered wheat-based diets had 5.0 and $6.2 \%$ higher $(P<0.05)$ jejunal and ileal DE, respectively, than those offered maizebased diets. In contrast, broilers offered maize-based diets had a $4.9 \%$ higher $(P<0.05)$ jejunal starch DC than those fed the wheat-based diet. The reduction in AA density in the maize-based diet trended to affect jejunal nitrogen DC $(P=0.10)$ and ileal DE $(P=0.10)$ with birds fed the PC diet having a 8.3 and $1.4 \%$ higher $(P<0.10)$ digestibility, respectively, than those receiving the $\mathrm{NC}$ diet. Likewise, AA density reduction in the wheat-based diet affected ileal nitrogen $(P=0.009)$ and jejunal starch $(P=0.01)$. Broilers offered the wheat-based NC had a $1.9 \%$ lower $(P<0.05)$ ileal nitrogen DC, but a $5.1 \%$ higher $(P<0.05)$ jejunal starch DC compared with those offered the PC. Moreover, the reduction AA density in the wheat-based diet trended to affect jejunal $\mathrm{DE}(P=0.06)$ with broilers receiving $\mathrm{PC}$ diet having a $6.6 \%$ higher $(P<0.10) \mathrm{DE}$ than those receiving the $\mathrm{NC}$ diet.

\section{Discussion}

The current study aimed to address whether protease, in combination with phytase and xylanase, would affect nitrogen and starch digestibility in the small intestine and performance of birds fed diets based either on wheat or maize. The results showed a significant cereal grain effect at $14 \mathrm{~d}$ of age, where the birds fed wheat-based diets had higher nitrogen and starch digestibility than their maizefed counterparts. Interestingly, protease supplementation affected starch digestibility coefficients of both wheat and maize diets. Protease inclusion significantly increased the ileal digestibility of starch by $1.5 \%$ (0.969 vs 0.954$)$ in the wheat-based diets but did not alter ileal starch digestibility in maize-based diets. These results manifested themselves as significantly increased ileal digestible energy in wheatbased diets as a consequence of protease supplementation. This was similar to the findings of Amerah et al. (2017), who observed that protease inclusion in maize soy-based NC diets (reduced amino acid and energy concentration) did not influence ileal nitrogen digestibility or DE, but significantly improved ileal starch digestibility coefficients by $0.7 \%$ (97.6 vs 96.9$)$. As starch is the major energy source in broiler diets, any improvement in its digestibility may have been an indication that protease supplementation spared dietary energy by indirectly disrupting the feed matrix, improving the digestibility of non-protein nutrients during this period of growth in chickens.
The study measured nutrient digestibility at $28 \mathrm{~d}$ of age, to determine if the effect of protease in combination with phytase and xylanase on nitrogen and starch digestion, as well as jejunal and ileal digestible energy values, changed. In general, wheat-based diets remained higher in digestibility than maize-based diets. Birds fed wheat-based diets presented higher DE values both in the jejunum and ileum, compared with maize-based diets. At $28 \mathrm{~d}$ of age, protease supplementation had a negative effect on ileal starch digestion in birds fed wheat-based diets but did not have an effect in those fed maize-based diets. This result was difficult to explain. Choct et al. (1999) demonstrated that wheat is probably the most variable cereal grain in terms of nutritive value for broiler chickens. Its energy value varies by $2-3 \mathrm{MJ} / \mathrm{kg}$ and its crude protein concentration by nearly $8 \%$ (10.8 to $18.3 \%)$. This variation is dependent on genetics and a number of environmental factors. Thus, it is possible that the wheat grain used in the current study was not of optimal quality. Of course, wheat contains higher levels of soluble non-starch polysaccharides than maize, which may increase digesta viscosity (Choct et al., 1996), thereby influencing nutrient digestibility to greater extent compared to maize-based diets. However, xylanase was included in all diets in the present study which should have negated any viscosity from wheat-based diets.

The significant cereal grain by protease interaction in nutrient digestibility was not apparent in the performance results. Indeed, protease supplementation of $\mathrm{NC}$ maize-based diets did not significantly influence FCR in comparison to unsupplemented NC diets. Liu et al. (2014) observed responses generated by an enzyme mix of protease, xylanase and amylase, which was dependent on the cereal grain used, with the enzyme combination having no influence on apparent ileal digestibility of starch or protein in maize-based diets. Additionally, the response to the enzyme supplementation was more pronounced in birds offered wheat-based diets than for maize-based diets, which was in agreement with the present study, albeit with a slight difference in enzymes tested. Despite the lack of response in nutrient digestibility, the enzyme cocktail significantly improved FCR of maize-based diets by $4.05 \%$. Cowieson and Ravindran (2008) reported that an enzyme combination of xylanase, amylase and protease significantly improved FCR in maize-based diets, and this was supported by increased distal ileal digestibility of energy and nitrogen, which was not observed in the present study.

The only key difference observed in bird performance was between the two cereal grains, with wheat being significantly inferior to maize. The lack of protease effect on performance was likely due to the protein and digestible amino acid levels in the dietary treatments being notably lower than the breeder recommendations in both the PC and NC treatments. The magnitude of reduction in dietary protein concentration may have caused an imbalance in the ratio 
Protease and cereal grain source

Table 7. Apparent nitrogen and starch digestibility and digestible energy in the jejunum and ileum of Ross $\times$ Ross 308 male broilers fed Maize- and wheat-based diets without or with supplemental protease at $28 \mathrm{~d}$ of age.1,2

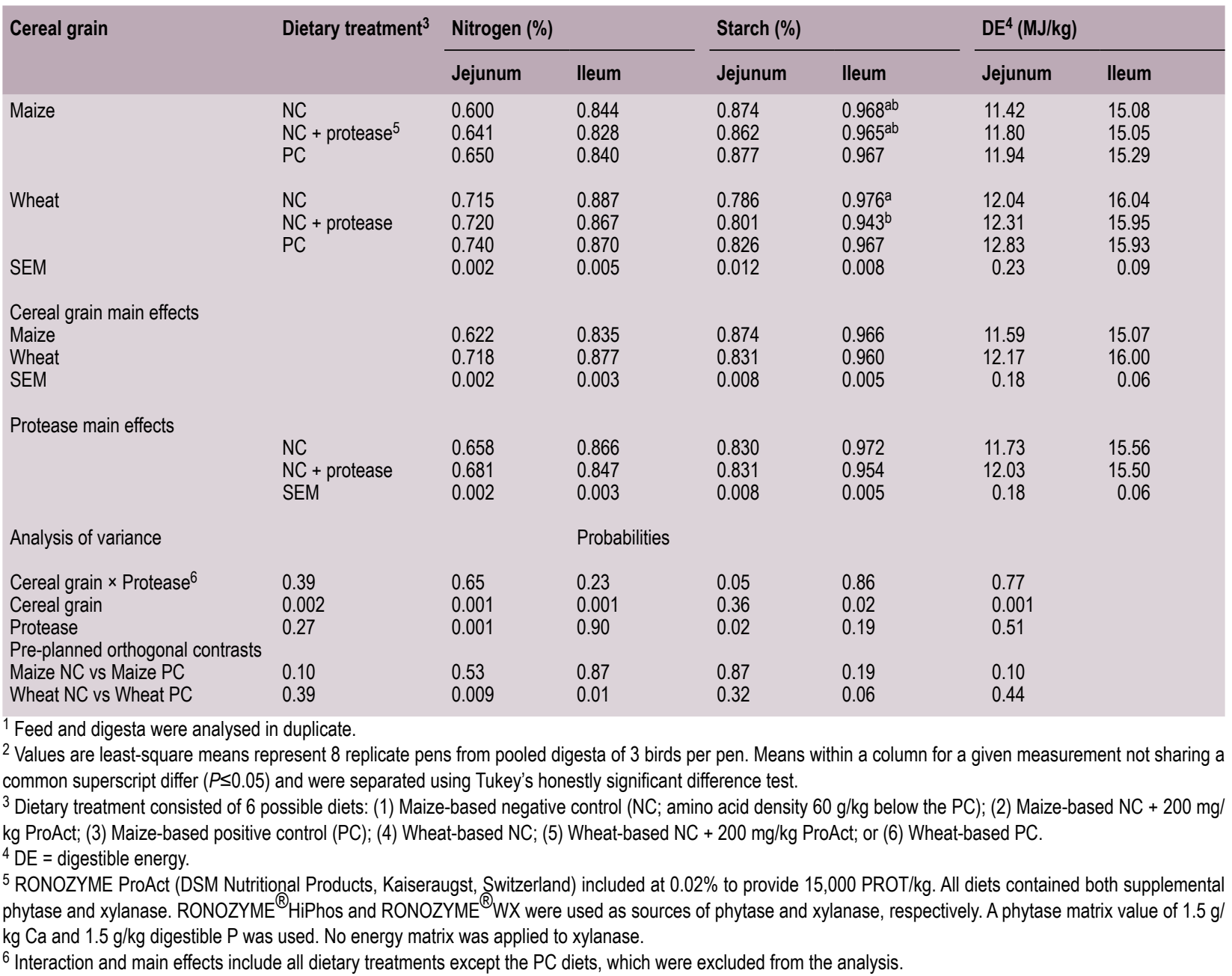

between AME and digestible amino acid and protein level, which was exacerbated further by the presence of xylanase and was not resolved by the protease. This was in agreement with Kidd and Tillman (2016), who stated that dietary lysine concentration and the ratio of other amino acids relative to lysine, non-essential amino acids and diet nitrogen content is, in part, responsible for variable responses seen in broiler performance when feeding low-protein diets. Thus, further research is warranted into bird performance response to protease in diets with low, but comparatively less extreme, amino acid concentrations. Moreover, Cowieson et al. (2020) stated that supplementation of synthetic amino acids or enzymes alone cannot counteract the multiple issues associated with feeding low-protein diets to broiler chickens. For example, they observed improved FCR in low-protein diets as a consequence of increasing dietary available phosphorus level. During the grower and finisher phase, the performance of birds fed the wheat-based diets lagged behind their maize-based counterparts, which were performing at or above the breed standard. In fact, the birds fed the wheat-based diets were nearly $163 \mathrm{~g}$ lighter ( $977 \mathrm{vs} 814 \mathrm{~g}$ ) at $28 \mathrm{~d}$ of age and $337 \mathrm{~g}$ lighter $(2,060 \mathrm{vs}$ $1,723 \mathrm{~g})$ at $35 \mathrm{~d}$ of age. Protease supplementation failed to alleviate this effect of cereal grain type on bird performance. It can be hypothesised that the differing AME to protein ratios between the wheat and maize-based diets, due to the comparatively higher protein level in wheat-based diets, may have been responsible for this observation, alongside the fundamental differences between the two grains.

Anomalies between digestibility results and performance data have been previously reported (Scott et al., 1998, 1999). In the current study, wheat attained greater ileal nitrogen digestibility and digestible energy than maize overall, and protease supplementation enhanced nitrogen and starch digestion at $14 \mathrm{~d}$ of age. There were a number of possible factors that may have dissociated digestibility and performance results. Firstly, fundamental physiochemical differences between the two grains would have affected diet composition and physical consistency, and the digestibility 
values were representative of a 'point in time' measurement, whereas performance represented cumulative changes. Secondly, the birds may have suffered from underlying health issues during the first week of life that were more pronounced when fed viscous grains, such as wheat. Indeed, one of the pre-disposing factors for necrotic enteritis is cereal grain type. Kaldhusdal and Hofshagen (1992) and Annett et al. (2002) showed marked differences in the incidence and severity of necrotic enteritis in broilers fed barley, wheat, or maize. Unfortunately, necrotic enteritis occurs in both clinical and subclinical forms, with the latter being devoid of clinical signs or even noticeable lesions but manifesting in reduced bird performance. In the present study, the one-day-old chicks were obtained from a hatchery more than $8 \mathrm{~h}$ drive from the research facilities. When the birds arrived, they appeared unthrifty and exhibited low BW. Consequently, the chicks were allowed $14 \mathrm{~d}$ to settle and adapt to the environment. Although performance was not the primary objective of this study, it was reported from 15 to $35 \mathrm{~d}$ of age.

\section{Conclusions}

Protease inclusion in reduced protein diets for broiler chickens may improve ileal starch digestibility; however, its efficacy may be dependent on the source of cereal-grain used and formulation strategy. Moreover, cereal grain type led to significant differences in performance of broiler chickens, with maize generating greater performance in this particular study.

\section{Acknowledgements}

The authors would like to thank DSM Nutritional Products for funding this study and for their support.

\section{Conflict of interest}

The authors declare no conflict of interest.

\section{References}

Amerah, A.M., Romero, L.F., Awati, A. and Ravindran, V., 2017. Effect of exogenous xylanase, amylase, and protease as single or combined activities on nutrient digestibility and growth performance of broilers fed corn/soy diets. Poultry Science 96: 807-816.

Angel, C., Saylor, W., Vieira, S. and Ward, N., 2011. Effects of a monocomponent protease on performance and protein utilization in 7-to 22-day-old broiler chickens. Poultry Science 90: 2281-2286.

Annett, C., Viste, J., Chirino-Trejo, M., Classen, H., Middleton, D. and Simko, E., 2002. Necrotic enteritis: effect of barley, wheat and corn diets on proliferation of Clostridium perfringens type A. Avian Pathology 31: 598-601.

Association of Official Agricultural Chemists (AOAC), 2005. Official methods of analysis. AOAC International, Gaithersburg, MD, USA.
Aviagen, 2014. ROSS 308 broiler: nutrition specifications. Aviagen, Huntsville, AL, USA. Available at: http://garantitavukculuk.com/ doc/Ross_nutrition_spec.pdf

Barletta, A., 2011. Introduction: current market and expected developments. In: Bedford, M. R. and Partridge, G.G. (eds.) Enzymes in farm animal nutrition. CABI, Wallingford, UK.

Bewley, J.D. and Black, M., 2013. Seeds: physiology of development and germination. Springer Science \& Business Media, New York, NY, USA.

Choct, M., Hughes, R. and Annison, G., 1999. Apparent metabolisable energy and chemical composition of Australian wheat in relation to environmental factors. Australian Journal of Agricultural Research 50: $447-452$

Choct, M., Hughes, R.J., Wang, J., Bedford, M., Morgan, A. and Annison, G., 1996. Increased small intestinal fermentation is partly responsible for the anti-nutritive activity of non-starch polysaccharides in chickens. British Poultry Science 37: 609-621.

Corzo, A., Fritts, C., Kidd, M. and Kerr, B., 2005. Response of broiler chicks to essential and non-essential amino acid supplementation of low crude protein diets. Animal Feed Science and Technology 118: 319-327.

Cowieson, A., Perez-Maldonado, R., Kumar, A. and Toghyani, M., 2020. Possible role of available phosphorus in potentiating the use of low-protein diets for broiler chicken production. Poultry Science 99: 6954-6963.

Cowieson, A. and Ravindran, V., 2008. Effect of exogenous enzymes in maize-based diets varying in nutrient density for young broilers: growth performance and digestibility of energy, minerals and amino acids. British Poultry Science 49: 37-44.

Cowieson, A.J. and Roos, F.F., 2013. Bioefficacy of a mono-component protease in the diets of pigs and poultry: a meta-analysis of effect on ileal amino acid digestibility. Journal of Applied Animal Nutrition 2: E13.

Cowieson, A.J. and Roos, F.F., 2016. Toward optimal value creation through the application of exogenous mono-component protease in the diets of non-ruminants. Animal Feed Science and Technology 221: 331-340.

Dean, D., Bidner, T. and Southern, L., 2006. Glycine supplementation to low protein, amino acid-supplemented diets supports optimal performance of broiler chicks. Poultry Science 85: 288-296.

Ferguson, N., Gates, R., Taraba, J., Cantor, A., Pescatore, A., Ford, M. and Burnham, D., 1998. The effect of dietary crude protein on growth, ammonia concentration, and litter composition in broilers. Poultry Science 77: 1481-1487.

Greenhalgh, S., Chrystal, P., Selle, P. and Liu, S., 2020. Reducedcrude protein diets in chicken-meat production: justification for an imperative. World's Poultry Science Journal 76: 537-548.

Hernández, F., López, M., Martínez, S., Megías, M., Catalá, P. and Madrid, J., 2012. Effect of low-protein diets and single sex on production performance, plasma metabolites, digestibility, and nitrogen excretion in 1-to 48-day-old broilers. Poultry Science 91: 683-692.

Kaldhusdal, M. and Hofshagen, M., 1992. Barley inclusion and avoparcin supplementation in broiler diets: 2. Clinical, pathological, and bacteriological findings in a mild form of necrotic enteritis. Poultry Science 71: 1145-1153. 
Kerr, B. and Kidd, M., 1999. Amino acid supplementation of lowprotein broiler diets: 1 . Glutamic acid and indispensable amino acid supplementation. Journal of Applied Poultry Research 8: 298-309.

Kidd, M. and Tillman, P., 2016. Key principles concerning dietary amino acid responses in broilers. Animal Feed Science and Technology 221: 314-322.

Liu, S., Cadogan, D., Péron, A., Truong, H. and Selle, P., 2014. A combination of xylanase, amylase and protease influences growth performance, nutrient utilisation, starch and protein digestive dynamics in broiler chickens offered maize-, sorghum-and wheatbased diets. Animal Production Science 55: 1255-1263.

National Health and Medical Research Council (NHMR), 2013. Australian code of practice for the care and use of animals for scientific purposes. NHMR, Canberra, ACT, Australia. Available at: https://www.nhmrc.gov.au/about-us/publications/australian-codecare-and-use-animals-scientific-purposes\#block-views-block-fileattachments-content-block-1.

SAS, 2015. SAS user's guide. SAS Inst. Inc., Cary, NC, USA.
Scott, T., Silversides, F., Classen, H., Swift, M. and Bedford, M., 1999. Prediction of the performance of broiler chicks from apparent metabolizable energy and protein digestibility values obtained using a broiler chick bioassay. Canadian Journal of Animal Science 79: 59-64.

Scott, T., Silversides, F., Classen, H., Swift, M., Bedford, M. and Hall, J., 1998. A broiler chick bioassay for measuring the feeding value of wheat and barley in complete diets. Poultry Science 77: 449-455.

Short, F., Gorton, P., Wiseman, J. and Boorman, K., 1996. Determination of titanium dioxide added as an inert marker in chicken digestibility studies. Animal Feed Science and Technology 59: 215-221.

Waldroup, P., Jiang, Q. and Fritts, C., 2005. Effects of supplementing broiler diets low in crude protein with essential and nonessential amino acids. International Journal of Poultry Science 4: 425-431.

Wang, H., Guo, Y. and Shih, J.C., 2008. Effects of dietary supplementation of keratinase on growth performance, nitrogen retention and intestinal morphology of broiler chickens fed diets with soybean and cottonseed meals. Animal Feed Science and Technology 140: 376-384. 
